\title{
Silver nanoparticle fabrication by laser ablation in polyvinyl alcohol solutions
}

\begin{abstract}
A laser ablation technique is applied for synthesis of silver nanoparticles in different concentrations of polyvinyl alcohol (PVA) aqueous solution. The ablation of high pure silver plate in the solution is carried out by a nanosecond Q-switched Nd:YAG pulsed laser. X-ray diffraction and transmission electron microscopy are implemented to explore the particles sizes. The effects of PVA concentrations on the absorbance of the silver nanoparticles are studied as well, by using a UV-vis spectrophotometer. The preparation process is carried out for deionized water as a reference sample. The comparison of the obtained results with the reference sample shows that the formation efficiency of nanoparticles in PVA is much higher and the sizes of particles are also smaller.
\end{abstract}

Keyword: Laser ablation; Silver nanoparticle; Polyvinyl alcohol 